\title{
Current-Pulse-Induced Switching of Asymmetric Spin Valves
}

\author{
P. BALÁZ̆ $\check{Z}^{a}$, M. GMitra ${ }^{b}$ AND J. BARNAŚ ${ }^{a, c}$ \\ ${ }^{a}$ Department of Physics, A. Mickiewicz University \\ Umultowska 85, 61-614 Poznań, Poland \\ ${ }^{b}$ Institute of Physics, P.J. Šafárik University \\ Park Angelinum 9, 04001 Košice, Slovakia \\ ${ }^{c}$ Institute of Molecular Physics, Polish Academy of Sciences \\ M. Smoluchowskiego 17, 60-179 Poznań, Poland
}

\begin{abstract}
Dynamical behavior of an asymmetric spin valve, $\mathrm{Co} / \mathrm{Cu} / \mathrm{Py}$ nanopillar sandwiched between $\mathrm{Cu}$ electrodes, subject to a spin-polarized current was investigated in the diffusive transport regime. Using numerical macrospin simulations, the dynamical diagrams of the sensing layer were constructed, and the possibilities of switching from one collinear state to the other were discussed. A simple switching scheme for asymmetric spin valves, based on a sequence of current and magnetic field pulses, was proposed and illustrated on an example.
\end{abstract}

PACS numbers: 72.25.Ba, 73.21.Ac

\section{Introduction}

Expectations of efficient computers and high-capacity miniaturized data storage devices motivate scientists since several decades to study ultrafast dynamical processes on mesoscopic scales. One of the possible ways to meet this expectation is based on the ideas evolved from the works of Slonczewski [1] and Berger [2]. They pointed out that current passing through a multilayer consisting of two ferromagnetic layers separated by a nonmagnetic spacer layer can exert a torque on the magnetic moments of the ferromagnetic layers. Indeed, it has been shown experimentally that spin-polarized current can switch the system between parallel (P) and antiparallel (AP) alignments of the layers' magnetization [3]. In practice, the three-layer structures (called also spin valves) are constructed in such a way that magnetic moment of one layer is fixed (reference layer) and that of the second layer is free to rotate (sensing layer).

If one applies a bias voltage, current flowing through the system can change the angle $\theta$ between magnetic moments of the reference and sensing layers. As predicted already by Slonczewski, the spin-transfer torque is actually dependent on $\theta$, and this angular dependence is one of the most important characteristics of spin valves. In the diffusive transport regime, this angular dependence for a symmetric spin valve can strongly differ from that for an asymmetric structure, as shown in Ref. [4]. Indeed, a spin valve whose magnetic layers are made of different materials can show a wavy-like dependence on $\theta$, which means that the spin torque vanishes and changes sign for a certain noncollinear configuration $\left(\theta=\theta_{\mathrm{c}}\right)$. Such a dependence implies that current can not only destabilize simultaneously both collinear configurations, but also can induce steady-state precessional modes even at zero magnetic field [5]. This is why asymmetric spin valves are interesting not only for the fundamental but also from the application point of view.

In this paper we focus on the asymmetric spin valve $\mathrm{Co}(8) / \mathrm{Cu}(10) / \mathrm{Py}(8)$ sandwiched between semi-infinite $\mathrm{Cu}$ electrodes. The valve consists of $8 \mathrm{~nm}$ thick reference layer made of cobalt and $8 \mathrm{~nm}$ thick sensing layer of permalloy, which are separated by $10 \mathrm{~nm}$ thick layer of copper. We have carried out numerical simulations of the current-induced spin dynamics for nanopillars of elliptically shaped cross-section with the major and minor axes equal to $130 \mathrm{~nm}$ and $60 \mathrm{~nm}$, respectively. We assume the Co film as the reference layer and consider current-induced dynamics of the Py layer. This is rather well justified as the anisotropy field of Py is relatively small and thus the corresponding critical current for excitation of Py layer is smaller than that for the Co layer.

\section{Model}

Time dependence of a unit vector $\hat{\boldsymbol{s}}=\left(s_{x}, s_{y}, s_{z}\right)$ along the spin moment of sensing layer in the macrospin model is described by the Landau-Lifshitz-Gilbert equation,

$$
\frac{\mathrm{d} \hat{\boldsymbol{s}}}{\mathrm{d} t}=-\left|\gamma_{\mathrm{g}}\right| \mu_{0} \hat{\boldsymbol{s}} \times \boldsymbol{H}_{\mathrm{eff}}-\alpha \hat{\boldsymbol{s}} \times \frac{\mathrm{d} \hat{\boldsymbol{s}}}{\mathrm{d} t}+\frac{\left|\gamma_{\mathrm{g}}\right|}{M_{\mathrm{s}} d} \boldsymbol{\tau},
$$

where $\gamma_{\mathrm{g}}$ is the gyromagnetic ratio, $\mu_{0}$ is the magnetic vacuum permeability, $M_{\mathrm{S}}$ is the saturated magnetization and $d$ is the sensing layer thickness. The Gilbert damping parameter is assumed to be constant, $\alpha=0.01$. The effective field $\boldsymbol{H}_{\text {eff }}$ includes contributions from external magnetic field $\left(H_{\text {ext }}\right)$, uniaxial magnetic anisotropy $\left(H_{\text {ani }}\right)$, and the demagnetization field $\left(\boldsymbol{H}_{\mathrm{dem}}\right) ; \boldsymbol{H}_{\mathrm{eff}}=$ $-H_{\mathrm{ext}} \hat{\boldsymbol{e}}_{z}-H_{\mathrm{ani}}\left(\hat{\boldsymbol{s}} \cdot \hat{\boldsymbol{e}}_{z}\right) \hat{\boldsymbol{e}}_{z}+\boldsymbol{H}_{\mathrm{dem}}$, where $\hat{\boldsymbol{e}}_{z}$ is the unit vector along the axis $z$. The last term in Eq. (1) stands for the torque due to spin transfer, $\boldsymbol{\tau}=\boldsymbol{\tau}_{\|}+\boldsymbol{\tau}_{\perp}$, which 
consists of in-plane, $\boldsymbol{\tau}_{\|}=a I \hat{\boldsymbol{s}} \times(\hat{\boldsymbol{s}} \times \hat{\boldsymbol{S}})$, and out-of-plane, $\boldsymbol{\tau}_{\perp}=b I \hat{\boldsymbol{s}} \times \hat{\boldsymbol{S}}$, components; here $\hat{\boldsymbol{S}}$ is a unit vector along the net spin of the reference layer, which is considered fixed and pointing along the $z$-axis. Apart from this, $I$ is the current density, which is positive when electrons flow from the reference ( $\mathrm{Co}$ ) towards sensing layer $(\mathrm{Py})$. The parameters $a$ and $b$ generally depend on the angle $\theta$ between the spin moments of the reference and sensing layers. These parameters have been calculated in the limit of diffusive transport, as described in Ref. [4].

In classical spin models, as the one assumed here, the torque vanishes in strictly collinear magnetic configurations, and no influence of the current can be then observed. To initiate the spin dynamics, spin moment of the sensing layer has to be tilted away from the quasi-equilibrium orientation (especially in the limit of zero temperature). Following this, we have considered $\hat{s}$ to be biased of about one degree $\left(\theta=1^{\circ}\right)$ away from the $z$-axis. The numerical analysis has been performed by integration of Eq. (1) using the Heun scheme [6] with an auto-adaptive time step.

\section{Numerical results and discussion}

We focus first on the numerical analysis of spin dynamics under a constant charge current density $I$. The corresponding dynamical diagrams are shown in Fig. 1. The diagrams show a time evolution of the $s_{z}$ spin component in the absence of external magnetic field (Fig. 1a) and with the magnetic field $H_{\text {ext }}=200$ Oe applied along the $z$-axis (Fig. 1b). The charge current in the diagrams is normalized to $I_{0}=10^{8} \mathrm{~A} \mathrm{~cm}^{-2}$. In the case considered here, i.e. wavy-like dependence of the spin-transfer torque, the current needed to destabilize the $\mathrm{P}$ (and also AP) configuration has to be of positive sign. Negative current, in turn, stabilizes both collinear configurations. The former case is of particular interest since for current exceeding a critical density both the $\mathrm{P}$ and AP states are unstable and precessional regime can be induced even in zero magnetic field. From Fig. 1a follows that the current density exceeding the value of $I / I_{0} \approx 0.6$ induces dynamics of the sensing layer. The spin is driven to the in-plane precessional (IPP) regime,

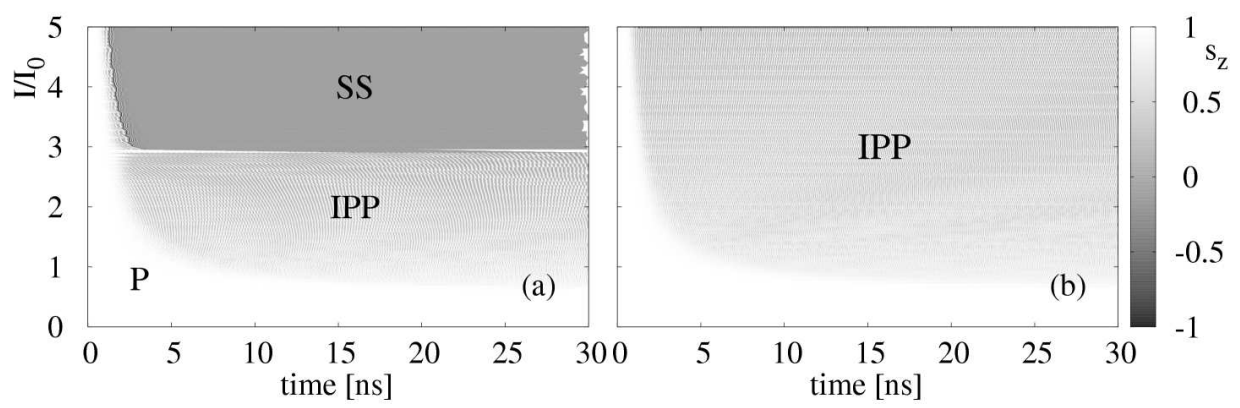

Fig. 1. Dynamical diagrams of the $\mathrm{Co}(8) / \mathrm{Cu}(10) / \mathrm{Py}(8)$ spin valve under a constant current of amplitude $I$ for (a) the case without external magnetic field, and (b) with the external magnetic field $H_{\text {ext }}=200$ Oe. The gray scale corresponds to the value of the $s_{z}$ component.

where $\hat{\boldsymbol{s}}$ precesses around the $z$-axis. The precessional regime is a result of the energy balance in which dissipation via Gilbert damping is compensated by the energy gain due to spin-transfer. When the current is switched off during these precessions, the spin of sensing layer ends either in $\mathrm{P}$ or AP state. This depends on the actual spin phase, i.e. on the actual basis of attraction of the trivial fixed points ( $\mathrm{P}$ and $\mathrm{AP}$ ) of the dynamics. When the current density surpasses the value $I / I_{0} \approx 3.0$, a new dynamical regime occurs. With the assistance of non-zero $\tau_{\perp}$, the spin is driven to the out-of-plane precessional (OPP) regime. The $\boldsymbol{\tau}_{\perp}$ overcomes then $\boldsymbol{\tau}_{\|}$which vanishes at $\theta=\theta_{\mathrm{c}}[5]$. The OPP regime is damped relatively fast and $\hat{s}$ asymptotically approaches the static state (SS) close to the $x$-axis $\left(s_{x} \approx \pm 1\right)$. These points are the stable points of dynamics. When current is turned off, the stable points become saddle points with separatrices connecting trivial fixed points. This finally leads to the spin relaxation towards either $\mathrm{P}$ or AP states. Therefore, the final state of the dynamics following after enough long current pulse is bistable.

Presence of an external magnetic field (pointing away from the SS state) lifts the bistability. For the case studied here, $H_{\text {ext }}=200$ Oe applied along the $z$-axis, the IPP regime occurs in the whole examined range of current densities, see Fig. 1b. When the current is switched off, the final state of the subsequent dynamics strongly depends on the oscillation phase at the time when current disappears. Due to the relatively short timescale of spin precession, the final state is again rather unpredictable. 


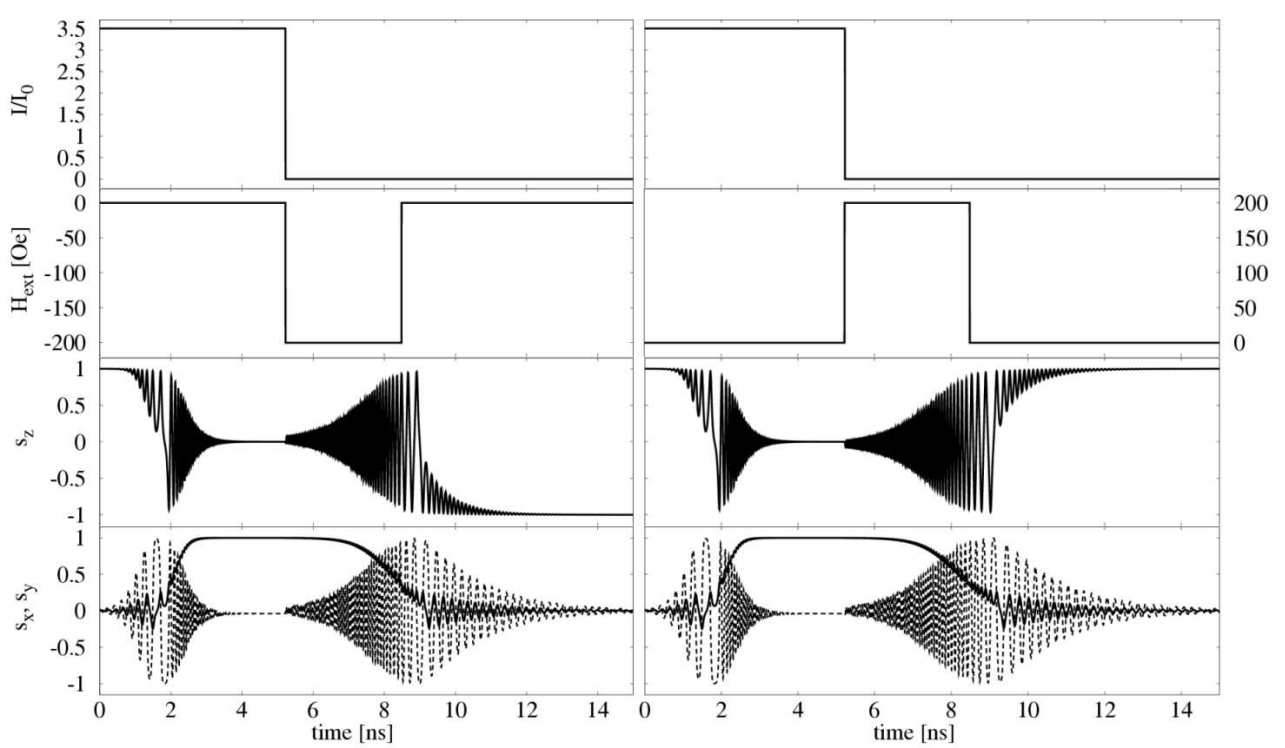

Fig. 2. The hybrid switching scheme based on the current and magnetic field pulses. From the top: the current pulse; magnetic field pulse; time evolution of spin component $s_{z}$; time evolutions of spin components $s_{x}$ (solid line) and $s_{y}$ (dot line). The left part describes switching to the AP state, while spin dynamics in the right part ends in the initial P state.

In order to overcome this bistability we propose a hybrid switching scheme based on the sequence of current and magnetic field pulses. The scheme is illustrated in Fig. 2, where both the pulses have been considered rectangular, with zero raising and falling times. We have used the current pulse of $I=3.5 I_{0}$ amplitude, which drives the system via the OPP to SS state in less than $4 \mathrm{~ns}$. Upon turning off the current we apply $4 \mathrm{~ns}$ pulse of 200 Oe magnetic field. This pulse allows to avoid the bistability and steer the spin into desired state. Generally, negative field pulse drives the system to the AP state (left part), whereas positive field pulse induces switching to the $\mathrm{P}$ state (right part).

\section{Summary and concluding remarks}

Using macrospin simulations, we have investigated spin dynamics of an asymmetric spin valve, induced by a spin current and external magnetic field. Such asymmetric structures show a wavy dependence of the spin transfer torque on the angle between magnetic moments of the reference and sensing layers. We have determined the critical currents needed to obtain steady-state precessions and static states in zero magnetic field. Because of the lack of predictable switching regime from $\mathrm{P}$ to AP state under a current pulse with/without a magnetic field, we proposed a simple switching scheme based on a current pulse followed by a magnetic field pulse.

The proposed switching scheme has been illustrated on a simple example. However, the durations and amplitudes of the applied pulses have not been optimized.
There are several factors which can influence the parameters of optimal switching pulse. For instance, the optimal pulse parameters are dependent on the pillar structure. Moreover, the temperature will also play an important role.

\section{Acknowledgments}

The work was supported by the EU through the Marie Curie Training network SPINSWITCH (MRTN-CT-2006-035327) as well as by Polish National Scientific Network ARTMAG, Slovak Ministry of Education as a research project MVTS POL/SR/UPJS07 and Slovak Grant Agency VEGA 1/0128/08.

\section{References}

[1] J. Slonczewski, J. Magn. Magn. Mater. 159, L1 (1996).

[2] L. Berger, Phys. Rev. B 54, 9353 (1996).

[3] M. Tsoi, A.G.M. Jansen, J. Bass, W.-C. Chiang, M. Seck, V. Tsoi, P. Wyder, Phys. Rev. Lett. 80, 4281 (1998); E.B. Myers, D.C. Ralph, J.A. Katine, R.N. Louie, R.A. Buhrman, Science 285, 867 (1999); J.A. Katine, F.J. Albert, R.A. Buhrman, E.B. Myers, D.C. Ralph, Phys. Rev. Lett. 84, 3149 (2000).

[4] J. Barnaś, A. Fert, M. Gmitra, I. Weymann, V.K. Dugaev, Phys. Rev. B 72, 024426 (2005).

[5] M. Gmitra, J. Barnaś, Appl. Phys. Lett. 89, 223121 (2006); Phys. Rev. Lett. 96, 207205 (2006); Phys. Rev. Lett. 99, 097205 (2007).

[6] P.E. Kloeden, E. Platen, Numerical Solution of Stochastic Differential Equations, Springer-Verlag, Berlin 1992. 